\title{
Versatile architecture of ultra-narrow band absorbing photonic nanostructure
}

\author{
Clément Verlhac ${ }^{1, *}$, Hasnaa El-Ouazzani ${ }^{1}$, Mathilde Makhsiyan ${ }^{1}$, Riad Haidar ${ }^{1,2}$, Jérôme Primot ${ }^{1}$, and Patrick \\ Bouchon $^{1, * *}$ \\ ${ }^{1}$ DOTA, ONERA, Université Paris-Saclay, F-91123 Palaiseau, France \\ ${ }^{2}$ École polytechnique, Département de Physique, 91128 Palaiseau, France
}

\begin{abstract}
Ultra narrow-band absorbing surfaces answer a growing demand of precise control of absorption spectra. Starting from a design of guided mode resonator (GMR), we present here a versatile architecture presenting resonances of very high quality factors (up to 10000) and spectrally adjustable. This design uses multiple slices of dielectrics to focus resonating field in the middle of a metallic waveguide, diminishing its extension in the metal and thus the loss rate of the propagating mode.
\end{abstract}

\section{Introduction}

Controlling the absorption spectrum of a surface - and its thermal emission as a consequence of Kirchhoff's law is crucial for a wide panel of applications ranging from filtering elements to light sources and thermal management. Metamaterials and more generally nanostructures offer the possibility of controlling the electromagnetic response of an effective medium by taking advantage of photonic and plasmonic responses of a sub-wavelength structuring of matter [1-3]. Since it has been shown such architectures were able to tune the impedance of a surface and adapt it to free space [4], using metamaterials to tailor spectral and angular absorption met a growing interest and numerous designs were proposed depending on the desired application [5]. Broadband absorbers are adapted to passive radiative cooling [6,7], thermophotovoltaics [8] and domestic light sources [9]. On the contrary narrowband absorbers are promising for gas sensing applications $[10,11]$ or as field enhancing structures since subwavelength narrow-band absorption is often synchronous with very high field concentration [12]. As we come to ultranarrow band absorbing structures, guided-mode resonators (GMR) are good candidates for they are known to present resonances with quality factors up to several thousands under certain circumstances [13]. GMR are based on a superficial diffraction grating that couples incident waves to guided modes propagating along the interface. Considering architectures with metal-dielectric waveguides, the narrowness, and thus the quality factor of the resonance, depends a lot on the propagation range of the guided mode and thus on its losses which are mainly subordinated to mode's penetration in the metallic components. Starting from this observation, this paper presents a multi-dielectric architecture of a GMR in which it is possible to control the

\footnotetext{
*e-mail: clement.verlhac@onera.fr

**e-mail: patrick.bouchon@onera.fr
}

bandwidth of the resonance by limiting the penetration in metal of the propagating mode.

\section{Description of the structure}

An usual architecture of a guided-mode resonator consists in the association of a metallic-dielectric waveguide and a metallic grating. The response of an usual one-dielectric structure is given by the red curve on fig.1(a) and its magnetic field map at resonance is depicted on fig.1(c). Under right condition of incidence, the grating diffracts incoming radiation along the surface which thus fuels a mode of the waveguide. If impedance matching is achieved, all the incoming energy is transferred into the guided-mode and then absorbed as the mode propagates. Under TM normal illumination, the structure resonates not far from $\lambda_{r}=\mathrm{p} * n$ where $\mathrm{p}$ is the period of the grating and $n$ the optical index of the dielectric. We propose to modify this design by subdividing the core of the waveguide into three slices of dielectrics where higher optical index is placed at the center and lower indices on the sides. As presented on fig. 1, this architecture resonates under the same conditions as the previous one. However, as depicted on the magnetic field map of fig.1(b), the resonating field is much more focused at the center of the waveguide. The loss rate of the guided mode is thus reduced, resulting in a 20 -fold reduction of the bandwidth of the peak. Both architectures are very close except for the width of gold's ribbon which is tuned to achieve perfect impedance matching.

This design presents a very good versatility. A modification of the geometrical parameters makes possible the tuning of the peak's wavelength throughout the whole 3-5 $\mu \mathrm{m}$ atmospheric transparency window and the adjustment of its quality factor from 100 up to 10000 .

To provide for an experimental proof of principle, a sample was designed and its angular and spectral reflectivity was measured using an adapted Fourier transform spectrometer. Even if, because of losses in the high index 
dielectric part, the quality factor of the resonance revealed itself to be not as high as expected $(Q \sim 80)$, numerical simulations and experimental curves are in very good agreement, proving the applicability of this concept.

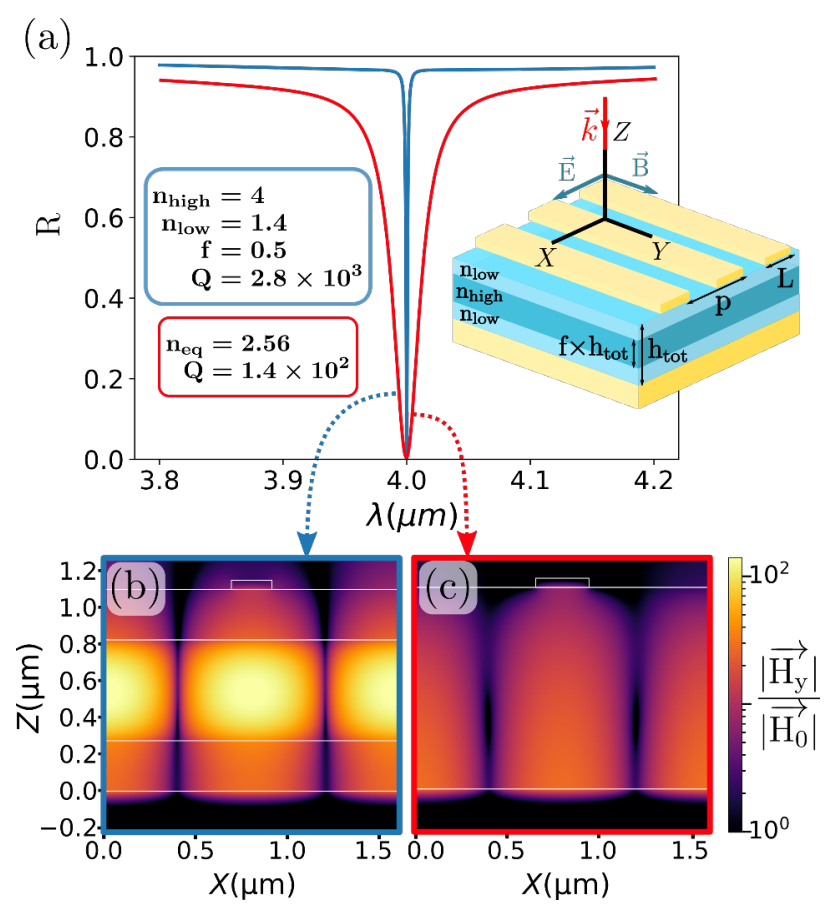

Figure 1. (a) General design of the structure in shown on insert. For both curves period $\mathrm{p}$ is set to $1.6 \mu \mathrm{m}$ and dielectric height $\mathrm{h}_{\text {tot }}$ to $1.1 \mu \mathrm{m}$. Blue and red curves respectively correspond to the responses of three- and one-dielectric design under TM normal illumination. For theses two architectures, the width of gold ribbon is respectively set to 220 and $290 \mathrm{~nm}$. (b) and (c) present the enhancement of magnetic field at the resonance $(4 \mu \mathrm{m})$ for both structures. The maximal values are respectively 140 and 27 .

\section{Conclusion}

This architecture seems quite promising in terms of versatility and potential applications. Quality factor of several thousand had already been observed with guided mode resonators but few nanophotonic concepts present as high values as depicted here. Losses in the metal becomes so low that, in practice, losses in the dielectric itself can limit the bandwidth. This apparent limitation opens the perspective of simultaneously concentrating losses and high field enhancement in the dielectric part of the waveguide which would make this design adapted to active optics such as exaltation of non-linear effects. Besides, the possibility of precisely choosing the spectral position of the resonance and its bandwidth makes this structure a promising candidate as thermal source for molecular sensing.

\section{References}

[1] V.G. Veselago, Soviet physics uspekhi 10, 509 (1968)

[2] D.R. Smith, W.J. Padilla, D. Vier, S.C. NematNasser, S. Schultz, Physical review letters 84, 4184 (2000)

[3] R.A. Shelby, D.R. Smith, S. Schultz, science 292, 77 (2001)

[4] N.I. Landy, S. Sajuyigbe, J.J. Mock, D.R. Smith, W.J. Padilla, Physical review letters 100, 207402 (2008)

[5] Y. Cui, Y. He, Y. Jin, F. Ding, L. Yang, Y. Ye, S. Zhong, Y. Lin, S. He, Laser \& Photonics Reviews 8, 495 (2014)

[6] E. Rephaeli, A. Raman, S. Fan, Nano letters 13, 1457 (2013)

[7] P.C. Hsu, C. Liu, A.Y. Song, Z. Zhang, Y. Peng, J. Xie, K. Liu, C.L. Wu, P.B. Catrysse, L. Cai et al., Science Advances 3, e1700895 (2017)

[8] A. Lenert, D.M. Bierman, Y. Nam, W.R. Chan, I. Celanović, M. Soljačić, E.N. Wang, Nature nanotechnology 9, 126 (2014)

[9] O. Ilic, P. Bermel, G. Chen, J.D. Joannopoulos, I. Celanovic, M. Soljačić, Nature nanotechnology 11, 320 (2016)

[10] A. Lefebvre, D. Costantini, G. Brucoli, S. Boutami, J.J. Greffet, H. Benisty, Sensors and Actuators B: Chemical 213, 53 (2015)

[11] H. Miyazaki, T. Kasaya, M. Iwanaga, B. Choi, Y. Sugimoto, K. Sakoda, Applied Physics Letters 105, 121107 (2014)

[12] P. Chevalier, P. Bouchon, J.J. Greffet, J.L. Pelouard, R. Haïdar, F. Pardo, Physical Review B 90, 195412 (2014)

[13] A. Sharon, S. Glasberg, D. Rosenblatt, A.A. Friesem, Journal of the Optical Society of America A 14, 588 (1997) 